\title{
Redo aortic valve replacement for prosthesis endocarditis in patients previously classified as high or prohibitive risk: a narrative review
}

\author{
Francesco Pollari ${ }^{1 \#}$, Renate Ziegler ${ }^{2 \#}$, Francesco Nappi ${ }^{3}$, Irena Großmann ${ }^{1}$, Jörg Steinmann ${ }^{2}$, \\ Theodor Fischlein ${ }^{1}$
}

${ }^{1}$ Department of Cardiac Surgery, ${ }^{2}$ Institute of Clinical Hygiene, Medical Microbiology and Infectiology, Klinikum Nürnberg - Paracelsus Medical University, Nuremberg, Germany; ${ }^{3}$ Department of Cardiac Surgery, Centre Cardiologique du Nord de Saint-Denis, Paris, France

Contributions: (I) Conception and design: F Pollari; (II) Administrative support: T Fischlein; (III) Provision of study materials or patients: None; (IV) Collection and assembly of data: F Pollari, R Ziegler, F Nappi; (V) Data analysis and interpretation: F Pollari, R Ziegler, T Fischlein; (VI) Manuscript writing: All authors; (VII) Final approval of manuscript: All authors.

\#These authors contributed equally to this work.

Correspondence to: Francesco Pollari, MD, PhD. Department of Cardiac Surgery, Klinikum Nürnberg - Paracelsus Medical University, Breslauer Strasse 201 - 90471 Nuremberg, Germany. Email: francesco.pollari@klinikum-nuernberg.de.

\begin{abstract}
Transcatheter aortic valve implantation (TAVI) and sutureless aortic valve replacement (Su-AVR) enabled in the last years many patients at high or prohibitive risk to be treated for their severe symptomatic aortic valve stenosis. As often happens in medicine, new techniques bring not only new hopes, but also new problems. In recent years, alongside the lengthening of the life of these patients treated with TAVI or SuAVR, cardiologists and cardiac surgeons have had to face the long-term complications associated with the implantation of these devices, such as the prosthetic infective endocarditis. The correct management of prosthesis valve endocarditis after TAVI or Su-AVR in high risk patients, and the possible role of surgery are a matter of debate because pushing the limits of the modern medicine and becoming a new challenge for cardiac surgeons of $21^{\text {st }}$ century. In this review, we summarized the incidence, characteristics and evidences for this new and controversial problem of the cardiovascular community. Moreover, we investigated the outcomes reported in literature of the conservative and the surgical strategy. Although the reported mortality rate of surgical treatment is high, seems not prohibitive, mostly if compared to conservative medical therapy. The collaborative exchange between cardiologist, cardiac surgeons, clinical microbiologists and expert of imaging is mandatory to face this challenge.
\end{abstract}

Keywords: Endocarditis team; infective endocarditis; transcatheter aortic valve implantation (TAVI); sutureless prosthesis; surgical high risk

Submitted Jun 21, 2020. Accepted for publication Jul 10, 2020.

doi: $10.21037 / \mathrm{atm}-20-4630$

View this article at: http://dx.doi.org/10.21037/atm-20-4630

\section{Introduction}

Transcatheter aortic valve implantation (TAVI) has changed the strategy for high risk/inoperable patients with severe aortic valve stenosis (1-3). Although the procedure has been recently extended to intermediate risk patients (4), and recent trials showed a short-term successful use in low risk $(5,6)$, nowadays the main and most common indication for TAVI remains the high or prohibitive risk patients. The typical TAVI population is therefore elderly, frail, having undergone multiple health care interventions, and consequently with a high risk of bacteremia and infected endocarditis (IE).

In the same period as TAVI emerged as alternative to standard aortic valve replacement (SAVR) for treatment 
of severe aortic valve stenosis, a new minimally invasive procedure was successfully applied: the aortic valve replacement with a sutureless prosthesis (Su-AVR) (7). The sutureless prosthesis (Perceval, LivaNova, Saluggia, Italy) resulted from a hybridization of TAVI and SAVR prostheses, bringing many advantages of both: on one hand, the sutureless prosthesis results any need of sutures, making the operation faster and allowing a minimally invasive approach (e.g., ministernotomy or minithoracotomy); on the other hand, the Su-AVR allows the removal of calcifications, that can be associated with many complications and device failure after TAVI (8-10). The characteristics of Su-AVR made it an attractive solution also for high-risk patients, putting itself as alternative to TAVI for those patients in the "grey zone" (7).

The common characteristics of patients that underwent TAVI or Su-AVR were also the high risk and frailty, a category of patients that in last years was not considered for surgery at all (11). The good results and the reduced invasivity of these two methods allowed a new category of patients, whom the advantages of surgery were previously denied, to receive a reliably treatment and to improve their life expectancy and quality.

As often happens in medicine, new techniques bring not only new hopes, but also new problems. In recent years, alongside the lengthening of the life of these patients treated with TAVI or Su-AVR, cardiologists and cardiac surgeons have had to face the long-term complications associated with the implantation of these devices, such as for example arrhythmogenic anomalies, anticoagulation management or the structural valve degeneration. Possibly the worst of the above-mentioned complication, the prosthetic infective endocarditis (IE) raised a big problem in the modern medicine. The treatment of IE requires a radical debridement of infected prosthetic material and surrounding tissue, which cannot be achieved due to percutaneous manipulations. IE in patients previously classified as high- or prohibitive risk became then a new challenge for cardiac surgeons of $21^{\text {st }}$ century.

In this review we will summarize the most recently evidences regarding incidence, characteristics and outcome of these challenging clinical scenario. Moreover, we will analyze and discuss some pragmatic suggestions for its management in the setting of the "Endocarditis Team".

We present the following article in accordance with the Narrative Review reporting checklist (available at http:// dx.doi.org/10.21037/atm-20-4630).

\section{Methods}

An English language literature review was performed through PubMed on June 2020 for any study evaluating the outcome after TAVI or Sutureless for severe aortic valve stenosis. The words employed in the search were "transcatheter aortic valve implantation", "transcatheter aortic valve replacement", "TAVI", "TAVR", "Sutureless aortic valve replacement", "Perceval", "Endocarditis". Since these technologies were widely used commercially after 2008, the literature search was limited to articles published since this year. Reference list of obtained articles were used to broaden the search. We applied the guidelines for Preferred Reporting Items for Systematic reviews (PRISMA).

\section{Incidence and characteristics of TAVI patients: results from big studies and our experience}

The efficacy of TAVI and non-inferiority in comparison with SAVR were firstly reported in the randomized Partner $(1,2)$ (using the balloon-expandable prosthesis "Sapien" [Edwards Lifescience]) and in CoreValve US Pivotal (3) (using the self-expandable prosthesis "Corevalve" [Medtronic]) trials, respectively in 2010 and 2011. Despite the relatively little number of samples, these trials are therefore - at the present time- - the source of evidence with the longest follow-up of regarding patients treated with TAVI prostheses. Other sources of data are the national and multicenter observational register, and single-center studies. We summarized the most relevant studies in the Table 1.

The incidence of PVE following TAVI, when reported, is about $1 \%$ during the first year. The result seems constant among registers and trials, independently from the chosen prosthesis (balloon, self, or mechanically expandable). Interestingly, the higher incidences are reported in singlecenter studies (up to $3 \%$ ), whereas the incidence remain around $1 \%$ in multicenter studies $(12,13)$. The Partner trial was structured in two cohorts: the 1A enrolling patients at high risk (randomized between SAVR and TAVI) and the $1 \mathrm{~B}$ enrolling inoperable patients (randomized between TAVI and medical therapy or valvuloplasty). Long term follow-up has been published only for 1A cohort, whereas for 1B only 1-year follow-up was reported. The biggest study population is reported by Regueiro and colleagues in a multicenter study from 47 centers worldwide (14). Among a cohort of 250 TAVI patients (from a total population accounting for 20,006 records) with a diagnosis of infective 
Table 1 Incidence of PVE following TAVI among literature

\begin{tabular}{|c|c|c|c|c|c|}
\hline Authors & Type of source & Country & Study period & $\begin{array}{l}\text { Study population } \\
\text { (number of patients) }\end{array}$ & Incidence of PVE after TAVI \\
\hline Mack MJ, et al. & $\begin{array}{l}\text { Randomized Multicenter trial } \\
\text { (PARTNER 1A) }\end{array}$ & $\begin{array}{l}\text { USA, Canada, } \\
\text { Germany }\end{array}$ & 2007-2009 & 348 & $\begin{array}{l}0.6 \% \text { during the first year; } \\
2 \% \text { at } 5 \text { years follow-up }\end{array}$ \\
\hline Leon MB et al. & $\begin{array}{l}\text { Randomized Multicenter trial } \\
\text { (PARTNER 1B) }\end{array}$ & $\begin{array}{l}\text { USA, Canada, } \\
\text { Germany }\end{array}$ & 2007-2009 & 179 & $1.1 \%$ during the first year \\
\hline Regueiro A, et al. & Multicenter Register & $\begin{array}{l}\text { North America, South } \\
\text { America, Europe }\end{array}$ & 2005-2015 & 20,006 & $1.1 \%$ per person-year \\
\hline $\begin{array}{l}\text { Amat-Santos IJ, } \\
\text { et al. }\end{array}$ & Multicenter Registry & $\begin{array}{l}\text { North America, South } \\
\text { America, Europe }\end{array}$ & 2007-2014 & 7,944 & $0.5 \%$ during the first year \\
\hline Moriyama N, et al. & Multicenter Registry & Finland & 2008-2017 & 2,130 & $0.7 \%$ \\
\hline Gilard M, et al. & Multicenter Registry & France & 2010-2012 & 4,201 & $0.8 \%$ \\
\hline Stortecky S, et al. & Multicenter Registry & Switzerland & 2011-2018 & 7,203 & $\begin{array}{l}1.29 \% \text { during the first year } \\
(1.48 \% \text { per person-year at first } \\
\text { year, } 0.4 \% \text { per person-year } \\
\text { after the first year) }\end{array}$ \\
\hline Mangner N, et al. & Single center & Germany & 2006-2014 & 1,820 & $\begin{array}{l}2.25 \% \text { during the first year, } \\
1.82 \% \text { per patient-year }\end{array}$ \\
\hline Olsen NT, et al. & Single center & Denmark & 2007-2014 & 509 & $\begin{array}{l}3.1 \% \text { during the first year, } \\
2.1 \% \text { per patient-year }\end{array}$ \\
\hline
\end{tabular}

endocarditis, they found an incidence of $1.1 \%$ per personyear (95\% CI, 1.1-1.4\%; median age, 80 years; $64 \%$ men). Median time from TAVR to infective endocarditis was 5.3 months [interquartile range (IQR), 1.5-13.4 months]. In addition, multivariate Cox regression analysis revealed as predictive factors younger age (HR: 0.97 per year; $95 \% \mathrm{CI}$ : 0.94 to 0.99 ), male sex (HR: $1.69 ; 95 \%$ CI: 1.13 to 2.52 ), diabetes mellitus (HR: 1.52; 95\% CI: 1.02 to 2.29), and moderate-to-severe aortic regurgitation (HR: 2.05; $95 \%$ CI: 1.28 to 3.28). Patients who developed PVE after TAVI had high rates of in-hospital mortality (36\%; 95\% CI: 30.0$41.9 \%$ ) and 2-year mortality (66.7\%, 95\% CI: 59.0-74.2\%).

The in-hospital mortality-rate in PVE after TAVI is $47 \%$, and $66 \%$ of patients died within the 1 year, thus accounting for the highest mortality-rate ever reported in the endocarditis field (15).

These findings are in line with our experience. Our institution (Cardiovascular center of Klinikum Nürnberg Paracelsus Medical University) started the TAVI-program at the end of 2009 and (up to May 2020) 1,550 patients were treated. We analyzed retrospectively the first 965 patients, for whom we have a completes follow-up. In this sample, we found 10 patients $(1 \%)$ were readmitted in our center for suspicious or confirmed diagnosis of PVE, according to the DUKES criteria (16). Of these, 4 cases occurred in the first year (mean follow-up 4.6 months) and the others 6 occurred after the first year (mean follow-up 1.97 years). Of the 4 early PVE, three cases were treated conservatively and died during the readmission, whereas one was successfully operated. Among the 6 patients readmitted because of late PVE, three died in-hospital and three were discharged (one of these against medical advice).

However, it should be not forgotten that TAVI patients are exposed not only to the risk of PVE, but also to infection of others native valves or permanent medical devices. Indeed, as these patients showed a rate of permanent pacemaker implantation significantly higher than surgery (ranging from 10-40\%) (9) are more prone to 
develop a right-side endocarditis. Unfortunately, current literature does not reflect this outcome and data are yet lacking.

\section{Incidence and characteristics of Sutureless patients: results from big studies and our experience}

Su-AVR is at the moment the most advanced evolution of the classical SAVR, capable to shorten the surgical times and to simplify the minimally invasive approach. Our group was pioneering in this technique (17).

Shrestha and colleagues reported in a single center study an incidence of 6\% (3 cases) PVE after Su-AVR among a small sample population of 50 high-risk patients (logistic Euroscore $20.4 \% \pm 10.7 \%$ ) (18). The reported mean followup time was $32.7 \pm 15.5$ months, but no detailed informations are reported about on-set of endocarditis.

The "Perceval Pivotal" was a trial conducted between 2009 and 2010 to assess the performance of the Perceval valve at 3 to 6 months after implantation in 150 patients at high surgical risk, aged 75 years or older, and requiring surgical intervention to replace the aortic valve. Freedom from valve explant was $100 \%$ at 5 -year follow-up evaluation. Aside from the patient dying from severe endocarditis and sepsis, a second patient suffered from prosthesis endocarditis, leading to re-hospitalization and complete resolution under antibiotic therapy on day 789 . Linearized rates (events per 100 patient-years) and actuarial probabilities of freedom from postoperative mortality and morbidity at 5 years for endocarditis were, 2.2 (95\% CI: $0.0-5.1$ ) and $90.7 \%$ respectively (19).

In a population of 243 that underwent combined SuAVR and coronary artery bypass grafting, no early PVE was observed whereas at follow-up there were 4 late explanations (1.35\%/patient-years) because of PVE (84, 169, 291, and 425 days after implantation, respectively). Although the mean logistic Euroscore was not extreme high $(12.1 \%)$, the mean age was $79.7 \pm 5.1$ years, and $48.1 \%$ of patients were 80 years or older (20). A similar finding was found in the "Perceval Cavalier trial" that reported $1.4 \%$ of endocarditis at 1-year follow-up in a similar population (21).

In our unreported single-center experience accounting 602 Su-AVR over 10 years, 7 patients experienced a PVE after Su-AVR at follow-up (1.1\%). Of these, 4 patients had early PVE (2, 4, 5 and 10 months), 1 after 3 years and 2 after 5 years. None of these patients had endocarditis on native valve (NVE) preoperatively. A Redo AVR was denied from 2 patients ( 2 and 5 months after Su-AVR), one of those died and the other survived after 6 weeks antibiotic therapy. Another patient with early PVE was successfully treated with antibiotics after discussion in the Endocarditis Team.

\section{Management of prosthesis endocarditis in high risk patients}

PVE has a higher mortality rate, increased complications rate, and a higher rate of treatment failure then patients with NVE even if infection occurs with the same pathogen. A multidisciplinary team approach is essential for optimal treatment of patients with endocarditis and current guidelines provide a Class IIa B recommendation for establishing an Endocarditis Team (22). This particularly applies for patients with a prosthetic valve or implantable cardiac device that are at higher risk for developing endocarditis, and for which the diagnosis according to Duke criteria could be challenging. For example, the echocardiographic criteria are often difficult to be interpreted, making other criteria-such as the clinical and microbiological-the critical ones. However, an implementation of Duke criteria using a multi-imaging approach with integration of echocardiography, computed tomography and 18F-fluorodeoxyglucose positron-emission tomography showed an excellent sensitivity (23).

An ideal Endocarditis Team requires the integration of experts from diverse medical fields for implementing appropriate care. Further on we focus on the role of infection prevention, microbiological diagnosis and antibiotic therapy in the management of prosthetic endocarditis (PVE) for high risk patients.

\section{The point of view of the microbiologist: infection prevention}

The best treatment of device-related infections is prevention. Despite less invasive nature of TAVI the rate of infective endocarditis after TAVI is comparable to that for surgical prosthetic valve endocarditis (14).

Some concerns have been raised about the adequacy of the sterile conditions in which the transcatheter valves are prepared and finally implanted. A current interim recommendation based on expert opinions, endorsed by the International Society for Cardiovascular Infectious Diseases (ISCVID), deals with infection control in TAVI (24). Owed that IE after TAVI is often related to the TAVI procedure 
itself and considering the high mortality rate after TAVI, the working group suggests to perform TAVI interventions in either designated catheterization laboratories or hybrid operating rooms. In both of them, the standards for sterile procedures (e.g., cleaning, room design, ventilation, limitation of area traffic, etc.) must be met as for other surgical procedures associated with implants. A recent analysis from the Swiss TAVI Registry on 7,203 patients who underwent TAVI at 15 hospitals in Switzerland showed that performing TAVI in a catheterization laboratory (in comparison with a hybrid room) was associated with an increased risk of PVE at multivariate analysis (OR 1.648, 95\% CI: $1.187-2.287 ; \mathrm{P}=0.003)(25)$.

Based on recommendations from WHO for SAVR (26), decolonization with nasal mupirocin ointment for 5 days may be considered if the patient is known to be a Staphylococcus aureus carrier or has a body mass index $>30 \mathrm{~kg} / \mathrm{m}^{2}$ and suffers from diabetes mellitus, although evidence for preventing IE in TAVI is lacking.

In a large registry reporting 250 cases, IE after TAVI was dominantly caused by Enterococcus species (24.6\%), especially in early IE, followed by Staphylococcus aureus (23.3\%), coagulase-negative staphylococci $(16.8 \%)$ and Viridans streptococci (6.9\%) (14). A recent systematic review confirmed this finding, showing that the most common causative organism is Enterococcus species (25.9\%) followed by Staphylococcus aureus $(16.1 \%)$ and coagulase-negative staphylococci (14.7\%) (27). One study (28) included patients from multicenter U.S. registry reported that causative pathogens were most commonly staphylococci strains (coagulase-negative staphylococci $25 \%$; Staphylococcus aureus $21 \%$ ) or enterococci (21\%). One-half of patients had previous procedure that was identified as the likely cause of bacteremia and an antibiotic prophylaxis was used in $59 \%$ of persons. As the commonly recommended antibiotic prophylaxis with an intravenous (IV) cephalosporin (cefazolin or cefuroxime) fails to cover enterococci prophylaxis with e.g., IV amoxicillin/ clavulanic acid 2.2 grams (single dose) preferably 0-60 minutes before intervention is being proposed. In cases where the TAVI procedure takes longer than two hours, a second dose of IV amoxicillin/clavulanic acid 2.2 grams should be administered. If the patient is known to be allergic to penicillin or colonized with penicillin-resistant Enterococcus species or methicillin-resistant Staphylococcus aureus intravenous vancomycin $(15 \mathrm{mg} / \mathrm{kg})$ is an alternative.

Evidence for the effectiveness of antibiotic prophylaxis for infective endocarditis is conflicting. Consequently, professional societies restricted the recommendation for antibiotic prophylaxis to high-risk patients only or even abandoned them completely (29-31). Patients with prosthetic cardiac valves (including transcatheter-implanted prostheses and homografts), with prosthetic material used for cardiac valve repair (such as annuloplasty rings and chords) and with previous IE should receive antibiotic prophylaxis for certain invasive dental procedures (32). Of note, gastrointestinal procedures such as colonoscopies no longer require antibiotic prophylaxis although several patients included in a review presented with PVE after such procedures (33). Amoxicillin is the drug of choice for oral prophylaxis, clindamycin in case of penicillin allergy and ampicillin, cefazolin or ceftriaxone intravenous for patients who are unable to tolerate an oral antibiotic.

\section{The point of view of the microbiologist: microbiological diagnosis}

Several studies report staphylococci, enterococci and streptococci as the most frequent causative microorganisms for IE after SAVR and TAVI $(33,34)$. Notably, a higher incidence of enterococci-positive blood cultures has been demonstrated in patients undergone transfemoral TAVI compared to the respective patients with postsurgical replacement. The use of transfemoral access in TAVI and the proximity of the groin with genitourinary/intestinal system constitute a strong predisposing factor for the frequent isolation of enterococci (14).

Identification of the causative microorganisms is essential for effective antibiotic therapy and mainly relies on blood culture diagnostic prior to antibiotic therapy. This applies particular for patients with prosthesis IE without valve surgery (e.g., ascending aorta replacement) or when lacking of additional molecular diagnostics on valve tissue.

The positive rate on blood culture becomes $90 \%$ or higher if blood specimens are collected before antibiotic treatments, but the positive rate on blood culture may decrease dramatically for some bacterial species if antibiotics have already been administered. Conventionally, three sets of blood cultures obtained from a peripheral vein, with each set including one aerobic and one anaerobic properly filled blood culture bottle, are collected. Multiple blood cultures at different time intervals increases sensitivity and enable a distinction between transient and persistent bacteremia. An aseptic technique for blood culture is mandatory since bacteria mostly considered as skin contaminants can also be the causative pathogens. The most common reason for so- 
called "culture-negative endocarditis" is due to concomitant or antecedent antibacterial therapy. In stable patients, recollection of blood cultures after an antibiotic-free period may be considered, also the ideal length of time needed off therapy is unknown. Organisms for which serologic tests have been shown to aid in the diagnosis of culture-negative endocarditis include Coxiella burnetii and Bartonella species. If the patient underwent valvular resection, culture of valve tissue, molecular testing, histopathology and staining of the resected valve is recommended. Culture of valve tissue suffer from low sensitivity and specify and molecular testing including broad-range bacterial PCR as well as specific PCR assays should be preferred (35). Obviously, in case of PVE histopathology and staining of prosthetic material are not possible, and therefore the surgeon should be careful to send to the laboratory also tissue adjacent the prosthesis in order to correct identify the pathogen.

\section{The point of view of the microbiologist: antibiotic therapy}

Data about treatment options and predictors of mortality in IE after TAVI are still rare and currently isolated medical management remains the most frequent strategy.

From two studies there are some pointers that surgery provided no significant mortality benefit compared with medical therapy only, despite the fact that patients treated by antibiotic therapy alone were older, had higher Society of Thoracic Surgeons scores and more often had severe chronic kidney disease than the operated patients $(14,36)$.

Standard IV drug therapy for PVE is similar as for hat of NVE but should last longer (6 weeks) $(22,37)$. Slow-growing and dormant microbes present in biofilms, displaying phenotypic tolerance towards most antimicrobials and relatively slow bactericidal activity of some antibiotics such as $\beta$-lactams and vancomycin justify the need for prolonged therapy to fully sterilize infected heart valves (38). For methicillin susceptible staphylococci (MSSA) a combination therapy of flucloxacillin with rifampicin and gentamicin is recommended, for streptococci penicillin $G$ or ampicillin or ceftriaxone. In case of methicillin resistant staphylococci (MRSA) or in B-lactam allergic patients vancomycin or daptomycin are considered alternatives. Enterococci are highly tolerant to antibiotic-induced killing, and eradication requires prolonged administration (as long as 6 weeks) of synergistic bactericidal combinations. Moreover, these microorganisms can be resistant to multiple drugs, including aminoglycosides, beta-lactams, vancomycin and linezolid.
Empirical treatment must cover the most common causative microorganisms estimated based on age, patients' medical background (such as dialysis), history of MRSA or VRE colonization, community-acquired infection or healthcareassociated infection.

In the design of dose regimens for the treatment of IE, it is important to fully optimize the pharmacokinetics and pharmacodynamics parameter for the selected antibiotic to increase the likelihood of success. While therapeutic drug monitoring (TDM) is established for aminoglycosides and vancomycin, data regarding $\beta$-lactam TDM and optimal dosing for patient with IE are limited (39). For the patients with positive blood cultures, blood cultures should be repeated within several days after the start of treatment (48 to 72 hours after the start of treatment) to confirm the effect of the treatment. Unresolved issues but with growing interest are the option of oral step down antibiotic therapy for IE with highly orally bioavailable antibiotics after clearing bacteremia and achieving clinical stability with intravenous regimens (40-42) and individualized long term suppressive therapy only in complicated prosthesis IE (43).

\section{Results of surgical treatment: what we learned until now}

Management of PVE after TAVI or Su-AVR is highly challenging. Unfortunately, especially for TAVIendocarditis, many of these patients were considered high or very high risk for surgery before undergoing valve intervention. If outcomes with antibiotic therapy alone are extremely poor, with in-hospital mortality ranging from $47 \%$ to $64 \%$, only a minority of patients $(<20 \%)$ underwent either open-heart surgery or a transcatheter valve-in-valve procedure in the studies to date (44).

In the previously cited work of Regueiro et al. (14), surgery was performed in $14.8 \%$ (95\% CI, 10.4-19.2\%) of patients during the infective endocarditis episode. Surgery during infective endocarditis hospitalization was associated with a reduced (but not significant) risk of in-hospital death (29.7\% ; 95\% CI: $15.0-44.4 \%$ for surgery vs. $37.1 \%$; $95 \%$ CI: $30.6-43.6 \%$ for no surgery).

Bjursten and colleagues analyzed 4,336 TAVI patients from the Sweden national registry. There were 103 patients identified as having PVE, where 54 were classified as definite IE according to the modified Duke criteria. The TAVI valve was deemed to be affected in $50 \%$ cases with either vegetation or abscess evident on echocardiography. Echocardiography also found vegetation on the mitral valve in $21 \%$ of cases. 
Open-heart surgery with aortic valve replacement (AVR) was only performed in 2 patients and pacemaker extraction was performed in 11 patients. In-hospital mortality was $16.8 \%$ and 1-year survival was $58.2 \%$ (45).

Amat-Santos and colleagues conducted a retrospective multicenter study in 21 centers in America and Europe. Among 53 cases of PVE after TAVI, a total of 46 patients $(86.8 \%)$ had at least 1 indication for valve intervention according to guidelines, and this included heart failure in 31 patients $(58.4 \%)$, septic shock in 11 patients $(26.8 \%)$, MRSA in 3 patients $(7.3 \%)$, structural complications in 16 patients $(30.2 \%)$, large vegetations $(>15 \mathrm{~mm})$ in 6 patients (14.6\%), and systemic emboli in 5 patients (12.2\%). Overall in-hospital mortality was reported in $25(47.2 \%)$ and mortality at follow-up in $13(24.5 \%)$ patients. New surgical intervention was performed in only 6 patients $(11.3 \%)$ : of these 4 survived and 2 died. In two cases, after antibiotic therapy a TAVI valve-in-valve was performed to treat the remaining structural deterioration (28).

A systematic review was conducted by Eisen et al. including 10 cases, 8 were from case reports and 2 cases were presented in congresses. Three (30\%) patients underwent surgery and $4(40 \%)$ patients died after postTAVI infective endocarditis (46). The major limitation of these systematic reviews was that most of the included articles were case reports and case series which may have precluded the real evaluation of the post-TAVI IE. Lastly, certain cases of post-TAVI infective endocarditis might not have been published leading to potential publication bias.

A recent report of the Society of Thoracic Surgeons reported the outcome of SAVR following TAVI in a study period between 2011 and 2015. Among the 123 patients who met the criteria, 12 were operated because of IE. In this population, operative mortality was $25 \%$ (47).

The challenge in operating a high- or prohibitive risk patient affected by PVE accounts not only for the poor clinical condition (determining the poor outcome) but also for relevant technical difficulties that the surgeon must face. Olsthoorn and colleagues reported their experience in treating a patient with previously CoreValve Evolut R $34 \mathrm{~mm}$ implantation. Difficulties were encountered with removing the stent frame adherent to the aorta. After excision of the valve, a neo-annulus was constructed with a pericardial patch and a biological stented valve was implanted. Additionally, a reversed sliding plasty of the mitral valve was necessary, as a result of an iatrogenic lesion of the anterior leaflet due to excision of the anchored skirt (48).

In our experience, we can report four cases of surgical intervention following PVE on TAVI (one) or Su-AVR (three). The PVE on TAVI occurred 6 months after index procedure and postoperative hospital staying was uneventful (49).

Among PVE after prior Su-AVR, four patients underwent Redo AVR: of those 1 occurred early (4 months) and died after operation. Of the others 3 late PVE, 1 died 5 days after redo AVR because of multiorgan failure and others 2 survived the surgical intervention without complications. In our direct experience, the Perceval prostheses did not show adherences of the stent with the aorta and the removal was technically simple (50). In the two patients who were operated after 5 years from the Su-AVR, some particularly strong adhesions were found at the annulus level, dissection was difficult and a patch reconstruction was necessary.

\section{Conclusions}

The correct treatment for PVE in high or prohibitive risk following TAVI or Su-AVR is a major problem of the modern cardiovascular medicine. The role of the Endocarditis Team is mandatory because of the challenging scenario, starting from the diagnosis up to the hospital discharge and follow-up. The role of surgery remains unclear because the lack of data. However, patients previously considered as high or prohibitive risk at the time of the first valve operation could have experienced overall functional recovery in the meantime, and subsequently become candidate for redo aortic valve replacement in case of PVE, as recently suggested by Jawitz and colleagues (47). The reported mortality rate after surgery is high, but if compared to conservative medical therapy is still acceptable. More data are needed to elucidate the role of the surgery, but a patient-tailored therapy under the supervision of the above-mentioned Endocarditis Team seems the most promising solution of the problem.

\section{Acknowledgments}

Funding: None.

\section{Footnote}

Provenance and Peer Review: This article was commissioned by the Guest Editors (Drs. Francesco Nappi, Christos Mihos, and Cristiano Spadaccio) for the series "Infective Endocarditis in the 21st Century" published in Annals of Translational Medicine. The article was sent for external peer 
review organized by the Guest Editors and the editorial office.

Reporting Checklist: The authors have completed the Narrative Review reporting checklist. Available at http:// dx.doi.org/10.21037/atm-20-4630

Conflicts of Interest: The authors have completed the ICMJE uniform disclosure form (available at http:// dx.doi.org/10.21037/atm-20-4630). The series "Infective Endocarditis in the 21st Century" was commissioned by the editorial office without any funding or sponsorship. FN served as the unpaid Guest Editor of the series and serves as an unpaid editorial board member of Annals of Translational Medicine from Feb 2019 to Jan 2021. TF is consultant for LivaNova. The other authors have no conflicts of interest to declare.

Ethical Statement: The authors are accountable for all aspects of the work in ensuring that questions related to the accuracy or integrity of any part of the work are appropriately investigated and resolved.

Open Access Statement: This is an Open Access article distributed in accordance with the Creative Commons Attribution-NonCommercial-NoDerivs 4.0 International License (CC BY-NC-ND 4.0), which permits the noncommercial replication and distribution of the article with the strict proviso that no changes or edits are made and the original work is properly cited (including links to both the formal publication through the relevant DOI and the license). See: https://creativecommons.org/licenses/by-nc-nd/4.0/.

\section{References}

1. Leon MB, Smith CR, Mack M, et al. PARTNER Trial Investigators. Transcatheter aortic-valve implantation for aortic stenosis in patients who cannot undergo surgery. $\mathrm{N}$ Engl J Med 2010;363:1597-607.

2. Smith CR, Leon MB, Mack MJ, et al. PARTNER Trial Investigators. Transcatheter versus surgical aorticvalve replacement in high-risk patients. $\mathrm{N}$ Engl J Med 2011;364:2187-98.

3. Gleason TG, Reardon MJ, Popma JJ, et al. 5-Year Outcomes of Self-Expanding Transcatheter Versus Surgical Aortic Valve Replacement in High-Risk Patients. J Am Coll Cardiol 2018;72:2687-96.

4. Baumgartner H, Falk V, Bax JJ, et al. 2017 ESC/EACTS
Guidelines for the management of valvular heart disease: The Task Force for the Management of Valvular Heart Disease of the European Society of Cardiology (ESC) and the European Association for Cardio-Thoracic Surgery (EACTS). Eur Heart J 2017;38:2739-91.

5. Mack MJ, Leon MB, Thourani VH, et al; PARTNER 3 Investigators. Transcatheter Aortic-Valve Replacement with a Balloon-Expandable Valve in Low-Risk Patients. N Engl J Med 2019;380:1695-705.

6. Popma JJ, Deeb GM, Yakubov SJ, et al; Evolut Low Risk Trial Investigators. Transcatheter Aortic-Valve Replacement with a Self-Expanding Valve in Low-Risk Patients. N Engl J Med 2019;380:1706-15.

7. Santarpino G, Pfeiffer S, Jessl J, et al. Sutureless replacement versus transcatheter valve implantation in aortic valve stenosis: a propensity-matched analysis of 2 strategies in high-risk patients. J Thorac Cardiovasc Surg 2014;147:561-7.

8. Pollari F, Dell'Aquila AM, Söhn C, et al. Risk factors for paravalvular leak after transcatheter aortic valve replacement. J Thorac Cardiovasc Surg 2019;157:14061415.e3.

9. Pollari F, Großmann I, Vogt F, et al. Risk factors for atrioventricular block after transcatheter aortic valve implantation: a single-centre analysis including assessment of aortic calcifications and follow-up. Europace 2019;21:787-95.

10. Pollari F, Hitzl W, Vogt F, et al. Aortic valve calcification as a risk factor for major complications and reduced survival after transcatheter replacement. J Cardiovasc Comput Tomogr 2020;14:307-13.

11. Iung B, Cachier A, Baron G, et al. Decision-making in elderly patients with severe aortic stenosis: why are so many denied surgery? Eur Heart J 2005;26:2714-20.

12. Mangner N, Woitek F, Haussig S, et al. Incidence, predictors, and outcome of patients developing infective endocarditis following transfemoral transcatheter aortic valve replacement. J Am Coll Cardiol 2016;67:2907-8.

13. Olsen NT, De Backer O, Thyregod HG, et al. Prosthetic valve endocarditis after transcatheter aortic valve implantation. Circ Cardiovasc Interv 2015;8:e001939.

14. Regueiro A, Linke A, Latib A, et al. Association Between Transcatheter Aortic Valve Replacement and Subsequent Infective Endocarditis and In-Hospital Death. JAMA 2016;316:1083-92.

15. Chourdakis E, Koniari I, Hahalis G, et al. Endocarditis after transcatheter aortic valve implantation: a current assessment. J Geriatr Cardiol 2018;15:61-5. 
16. Durack DT, Lukes AS, Bright DK. New criteria for diagnosis of infective endocarditis: utilization of specific echocardiographic findings. Duke Endocarditis Service. Am J Med 1994;96:200-9.

17. Fischlein T, Pfeiffer S, Pollari F, et al. Sutureless Valve Implantation via Mini J-Sternotomy: A Single Center Experience with 2 Years Mean Follow-up. Thorac Cardiovasc Surg 2015;63:467-71.

18. Shrestha M, Maeding I, Höffler K, et al. Aortic valve replacement in geriatric patients with small aortic roots: are sutureless valves the future? Interact Cardiovasc Thorac Surg 2013;17:778-82.

19. Meuris B, Flameng WJ, Laborde F, et al. Five-year results of the pilot trial of a sutureless valve. J Thorac Cardiovasc Surg 2015;150:84-8.

20. Shrestha M, Folliguet TA, Pfeiffer S, et al. Aortic valve replacement and concomitant procedures with the Perceval valve: results of European trials. Ann Thorac Surg 2014;98:1294-300.

21. Fischlein T, Meuris B, Hakim-Meibodi K, et al. The sutureless aortic valve at 1 year: A large multicenter cohort study. J Thorac Cardiovasc Surg 2016;151:1617-1626.e4.

22. Habib G, Lancellotti P, Antunes MJ, et al. 2015 ESC Guidelines for the management of infective endocarditis: the Task Force for the Management of Infective Endocarditis of the European Society of Cardiology (ESC). Eur Heart J 2015;36:3075-128.

23. Salaun E, Sportouch L, Barral PA, et al. Diagnosis of Infective Endocarditis After TAVR: Value of a Multimodality Imaging Approach. JACC Cardiovasc Imaging 2018;11:143-6.

24. Conen A, Stortecky S, Moreillon P, et al. A review of recommendations for infective endocarditis prevention in patients undergoing transcatheter aortic valve implantation. EuroIntervention 2020;EIJ-D-19-00993. doi:10.4244/EIJ-D-19-00993.

25. Stortecky S, Heg D, Tueller D, et al. Infective Endocarditis After Transcatheter Aortic Valve Replacement. J Am Coll Cardiol 2020;75:3020-30.

26. Global guidelines for the prevention of surgical site infection, second edition, ISBN 978-92-4-155047-5, World Health Organization 2018.

27. Khan A, Aslam A, Satti KN, et al. Infective endocarditis post-transcatheter aortic valve implantation (TAVI), microbiological profile and clinical outcomes: A systematic review. PLoS One 2020;15:e0225077.

28. Amat-Santos IJ, Messika-Zeitoun D, Eltchaninoff H, et al. Infective endocarditis after transcatheter aortic valve implantation: results from a large multicenter registry. Circulation 2015;131:1566-74.

29. Wilson W, Taubert KA, Gewitz M, et al. Prevention of infective endocarditis: guidelines from the American Heart Association: a guideline from the American Heart Association Rheumatic Fever, Endocarditis, and Kawasaki Disease Committee, Council on Cardiovascular Disease in the Young, and the Council on Clinical Cardiology, Council on Cardiovascular Surgery and Anesthesia, and the Quality of Care and Outcomes Research Interdisciplinary Working Group. Circulation 2007;116:1736-54.

30. Thornhill MH, Dayer MJ, Forde JM, et al. Impact of the NICE guideline recommending cessation of antibiotic prophylaxis for prevention of infective endocarditis: before and after study. BMJ 2011;342:d2392.

31. Habib G, Hoen B, Tornos P, et al. Guidelines on the prevention, diagnosis, and treatment of infective endocarditis (new version 2009): the Task Force on the Prevention, Diagnosis, and Treatment of Infective Endocarditis of the European Society of Cardiology (ESC). Endorsed by the European Society of Clinical Microbiology and Infectious Diseases (ESCMID) and the International Society of Chemotherapy (ISC) for Infection and Cancer. Eur Heart J 2009;30:2369-413.

32. Nishimura RA, Otto CM, Bonow RO, et al. 2017 AHA/ ACC focused update of the 2014 AHA/ACC guideline for the management of patients with valvular heart disease: a report of the American College of Cardiology/American Heart Association Task Force on Clinical Practice Guidelines. Circulation 2017;135:e1159-e1195.

33. Amat-Santos IJ, Ribeiro HB, Urena M, et al. Prosthetic valve endocarditis after transcatheter valve replacement: a systematic review. JACC Cardiovasc Interv 2015;8:334-46.

34. Summers MR, Leon MB, Smith CR, et al. Prosthetic Valve Endocarditis After TAVR and SAVR: Insights From the PARTNER Trials. Circulation 2019;140:1984-94.

35. Liesman RM, Pritt BS, Maleszewski JJ, et al. Laboratory Diagnosis of Infective Endocarditis. J Clin Microbiol 2017;55:2599-608.

36. Mangner N, Leontyev S, Woitek FJ, et al. Cardiac Surgery Compared With Antibiotics Only in Patients Developing Infective Endocarditis After Transcatheter Aortic Valve Replacement. J Am Heart Assoc 2018;7:e010027.

37. Baddour LM, Wilson WR, Bayer AS, et al. Infective Endocarditis in Adults: Diagnosis, Antimicrobial Therapy, and Management of Complications: A Scientific Statement for Healthcare Professionals From the American Heart Association Circulation 2015;132:1435-86. 
38. Di Domenico EG, Rimoldi SG, Cavallo I, et al. Microbial biofilm correlates with an increased antibiotic tolerance and poor therapeutic outcome in infective endocarditis. BMC Microbiol 2019;19:228.

39. Öbrink-Hansen K, Wiggers H, Bibby BM, et al. Penicillin G Treatment in Infective Endocarditis Patients - Does Standard Dosing Result in Therapeutic Plasma Concentrations?. Basic Clin Pharmacol Toxicol 2017;120:179-86.

40. Spellberg B, Chambers HF, Musher DM, et al. Evaluation of a paradigm shift from intravenous antibiotics to oral step-down therapy for the treatment of infective endocarditis: a narrative review. JAMA Intern Med 2020;180:769-77.

41. Iversen K, Ihlemann N, Gill SU, et al. Partial oral versus intravenous antibiotic treatment of endocarditis. N Engl J Med 2019;380:415-24.

42. Bundgaard H, Ihlemann N, Gill SU, et al. Long-term outcomes of partial oral treatment of endocarditis. N Engl J Med 2019;380:1373-4.

43. Lechner AM, Pretsch I, Hoppe U, et al. Successful longterm antibiotic suppressive therapy in a case of prosthetic valve endocarditis and a case of extensive aortic and subclavian graft infection. Infection 2020;48:133-6.

44. Cahill TJ, Baddour LM, Habib G, et al. Challenges in

Cite this article as: Pollari F, Ziegler R, Nappi F, Großmann I, Steinmann J, Fischlein T. Redo aortic valve replacement for prosthesis endocarditis in patients previously classified as high or prohibitive risk: a narrative review. Ann Transl Med 2020;8(23):1629. doi: 10.21037/atm-20-4630
Infective Endocarditis. J Am Coll Cardiol 2017;69:325-44.

45. Bjursten H, Rasmussen M, Nozohoor S, et al. Infective endocarditis after transcatheter aortic valve implantation: a nationwide study. Eur Heart J 2019;40:3263-9.

46. Eisen A, Shapira Y, Sagie A, et al. Infective endocarditis in the transcatheter aortic valve replacement era: comprehensive review of a rare complication. Clin Cardiol 2012;35:E1-E5.

47. Jawitz OK, Gulack BC, Grau-Sepulveda MV, et al. Reoperation After Transcatheter Aortic Valve Replacement: An Analysis of the Society of Thoracic Surgeons Database. JACC Cardiovasc Interv 2020;13:1515-25.

48. Olsthoorn JR, Lam K, Verberkmoes NJ. Endocarditis after transcatheter aortic valve replacement; a new nightmare in cardiac surgery. J Card Surg 2019;34:1420-1.

49. Santarpino G, Fischlein T, Pfeiffer S. Prosthetic valve endocarditis 6 months after transcatheter aortic valve implantation. G Ital Cardiol (Rome) 2013;14:138-40.

50. Di Bacco L, Pfeiffer S, Fischlein TJM, et al. Rapid Explantation of Rapid-Deployment Sutureless Valve in Case of Acute Endocarditis: How to Remove Safely Sutureless Perceval S Prostheses. Innovations (Phila) 2017;12:483-5. 